\title{
IL-6, PF-4, sCD40 L, and homocysteine are associated with the radiological progression of cerebral small-vessel disease: a 2-year follow-up study
}

This article was published in the following Dove Press journal: Clinical Interventions in Aging

Jacek Staszewski'

Renata Piusińska-Macoch'

Bogdan Brodacki'

Ewa Skrobowska ${ }^{2}$

Adam Stępień'

'Clinic of Neurology, ${ }^{2}$ Department of Radiology, Military Institute of Medicine, Warsaw, Poland
Correspondence: Jacek Staszewski Clinic of Neurology, Military Institute of Medicine, Szaserow 128, Warsaw 04-I4I, Poland

Tel +48 26I 816445

Fax +48228106100

Email jstaszewski@wim.mil.pl

\begin{abstract}
Background: Endothelial dysfunction (ED) is involved in the pathogenesis of cerebral small vessel disease (SVD), however, it is not clear if specific biomarkers related to ED are associated with radiological progression of SVD.

Methods: A single-center, prospective cohort study was conducted among consecutive, adult patients with SVD. Logistic regression was used to analyze the association of each baseline biomarker (highest vs lowest tertile) and the MRI radiological outcome after 2 years. The mean Z-score for vascular inflammation (VI) combined soluble intercellular cell adhesion molecule-1 (sICAM-1), soluble platelet selectin (sP-selectin), CD40 ligand (sCD40 L), platelet factor-4 (PF-4) and homocysteine; Z-score for systemic inflammation (SI) combined high-sensitivity C-reactive protein (hsCRP), interleukin-1 $\alpha$ and -6 (IL-1 $\alpha$ and IL-6, respectively) and tumor necrosis factor- $\alpha$ (TNF- $\alpha)$.
\end{abstract}

Results: The study group comprised 123 patients (age, mean \pm SD: $72.2 \pm 8$ years, $49 \%$ females), with lacunar stroke $(n=49)$, vascular dementia $(n=48)$, and vascular parkinsonism $(n=26)$. Moreover, 34.9\% patients experienced radiological progression, 43\% had progression of isolated white matter lesions (WMLs), 23.2\% had new lacunes, and 34.8\% had both WMLs progression and new lacunes. After adjustment for confounders (age, sex, blood pressure, MRI lesions load), the PF-4 (OR; 95\% CI 5.5; 1.5-21), sCD40L (4.6; 1.1-18.6), IL-6 (7.4; 1.48-37), Z-score for VI (4.5; 1.1-18.6), and, marginally, homocysteine (4.1; 0.99-17) were associated with the risk of any radiological progression; further, homocysteine $(2.4 ; 1.4-14), \mathrm{Z}$-score for SI $(2.1 ; 1.2-14)$ and, marginally, IL-6 (6.0; 0.95 -38) were related to the development of new lacunes; PF-4 (7.9; 1.6-38) and, marginally, the Z-score for VI (4.2; 0.9-19.5) were correlated with the risk of WMLs progression. Additional adjustment for clinical SVD manifestations did not significantly alter the results.

Conclusion: The data supports the concept that ED modulates the radiological progression of SVD and WMLs and lacunes are associated with different inflammatory markers.

Keywords: cerebral small-vessel disease, radiological progression, IL-6, PF-4, sCD40 L, homocysteine

\section{Introduction}

Cerebral small-vessel disease (SVD) is one of the most important and most common vascular diseases of the brain. ${ }^{1}$ It is responsible for the majority of lacunar stroke (LS) cases, vascular dementia (VaD), and parkinsonism (VaP). The main features of MRI are interrelated and include white matter lesions (WMLs), lacunar infarcts (LIs), enlarged perivascular spaces (PVS), microbleeds (MBs), and brain atrophy. ${ }^{2}$ These 
changes frequently progress over time, resulting in clinical deterioration. Recent studies have provided evidence that the disease is mostly non-atheromatous because the vascular risk factors can explain only a minority of the variance among the radiological features. ${ }^{3}$ Endothelial dysfunction (ED) is currently considered the most important mechanism of the structural and functional brain-vessel alterations related to SVD. The course and predictors of radiological progression in symptomatic SVD, especially in the rarely studied VaP or $\mathrm{VaD}$, are not well established. We have recently found a relationship among hemostatic markers - namely, fibrinogen, tissue factor, and beta-thromboglobulin - and the risk of radiological progression in patients with $\mathrm{LS}, \mathrm{VaD}$, and $\mathrm{VaP}{ }^{4}$ Considering that ED is involved in the pathogenesis of SVD, we hypothesized that some blood-derived markers of ED and the inflammatory cascade are linked to the risk of radiological progression in patients older than 60 years with different SVD manifestations.

\section{Materials and methods}

A single-center, prospective cohort study, with a 2-year radiological follow-up, was conducted as a part of the SHEFSVD Study. ${ }^{5}$ The detailed protocol and methods have been thoroughly described elsewhere. Briefly, the study group comprised 123 consecutive patients $>60$ years who were enrolled between December 2011 and June 2014: 49 had recent LS (39.8\%), 48 had $\mathrm{VaD}(39 \%)$, and 26 had $\mathrm{VaP}$ $(21.1 \%)$ - all were considered a result of sporadic SVD and diagnosed according to the typical radiological and clinical criteria (LS, according to the Oxfordshire Community Stroke Project; VaP and VaD according to the Hurtig or the International Workshop of the National Institute of Neurological Disorders and Stroke and the Association Internationale pour la Recherche et l'Enseignement en Neurosciences (NINDS-AIREN) criteria). ${ }^{6-8}$ Patients with an acute or chronic infectious process, malignancy, rheumatologic disease, significant stenosis ( $\geq 50 \%$ ) of a major extracranial or intracranial artery, atrial fibrillation, non-SVD-related WMLs, life expectancy of $<6$ months, and MRI contraindications were excluded.

Based on a priori knowledge of biological relevance, we investigated a set of markers related to ED and systemic inflammation (SI): high-sensitivity C-reactive protein (hsCRP), interleukin-1 alpha and -6 (IL-1 $\alpha$ and IL-6, respectively), tumor necrosis factor- $\alpha$ (TNF- $\alpha)$; and ED and vascular inflammation (VI): soluble intercellular cell adhesion molecule-1 (sICAM-1), soluble platelet selectin (sP-selectin), CD40 ligand (sCD40 L), platelet factor-4 (PF-4), and homocysteine. ${ }^{9}$ Plasma was isolated from overnight fasting blood samples, and biomarkers were assessed using commercially available ELISA kits according to the manufacturer's protocol (Bio-Source Europe, Nivelles, Belgium); duplicate readings were recorded, and the intra-assay coefficients of variation were $<10 \%$. All assays were conducted by individuals blinded to the study status. In this article, when a compound or metabolite is mentioned, it is the serum or plasma concentration that is stated. To prevent any confounding from the hyperacute-phase responses, all LS patients underwent study procedures at least 2 weeks (mean 17.5 \pm 3.8 days) after their index strokes.

MRI examinations were conducted at baseline and after

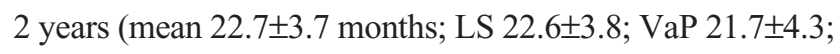
and $\mathrm{VaD} 23.2 \pm 3.3$ months, $p=0.2$ ), and MRI findings were categorized according to the Standards for Reporting Vascular Changes on Neuroimaging (STRIVE) guidelines as the reference standard. ${ }^{10}$ Images were rated for the presence of LIs, periventricular (pWMLs) or deep WMLs (dWMLs), MBs, and PVS according to the recommended visual SVD and Fazekas scales. ${ }^{11}$ Mild WMLs (Grade 1) were defined as punctate lesions in the deep white matter, with a maximum diameter of $9 \mathrm{~mm}$ for a single lesion and $20 \mathrm{~mm}$ for grouped lesions. Moderate WMLs (Grade 2) were early confluent lesions of 10 to $20 \mathrm{~mm}$ single lesions as well as grouped lesions of any diameter measuring $>20 \mathrm{~mm}$ with nothing more than connecting bridges between the individual lesions. Severe WMLs (Grade 3) were single lesions or confluent areas of hyperintensity of $\geq 20 \mathrm{~mm}$ of any diameter. All subjects had at least Fazekas Grade 1 WMLs. One point was awarded on the SVD scale if (early) there were confluent deep WMLs (Fazekas scores 2 and 3) or irregular periventricular hyperintensities extending into the deep white matter (Fazekas Score 3). A point was awarded if moderate to extensive $(10-25$ or $>25)$ enlarged PVS were present. Finally, the presence of each of the above markers produced a minimum score of 0 and a maximum of 4 - representing the total MRI load of SVD. Radiological progression was defined as an increase in WMLs (WML

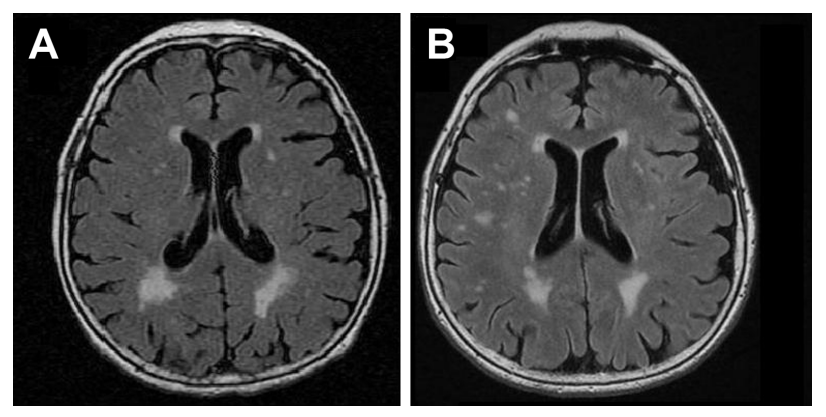

Figure I MRI axial T2-weighted-Fluid-Attenuated Inversion Recovery (T2-FLAIR) images at the level of lateral ventricles showing progression of deep white matter lesions at the 2-year follow-up (A, initial scan; $\mathbf{B}$, follow-up). 
Table I Baseline characteristics of the study population with and without radiological progression

\begin{tabular}{|c|c|c|c|c|c|c|c|}
\hline Variable & $\begin{array}{l}\text { No } \\
\text { progression }\end{array}$ & $\begin{array}{l}\text { Radiological } \\
\text { progression }\end{array}$ & $p$-value* & & $\begin{array}{l}\text { No } \\
\text { progression }\end{array}$ & $\begin{array}{l}\text { Radiological } \\
\text { progression }\end{array}$ & $p$-value* \\
\hline n (\%) & $80(65)$ & $43(35)$ & - & SVD score & $2.22(1)$ & $2.14(0.9)$ & 0.6 \\
\hline SVD manifestation; $N(\%)$ LS & $37(46.3)$ & $12(27.9)$ & 0.09 & SVD score 0 & $2(2.4)$ & 0 & 0.4 \\
\hline $\mathrm{VaD}$ & $26(32.5)$ & $22(5 \mid .2)$ & & 1 & $23(28.8)$ & $12(27.9)$ & \\
\hline $\mathrm{VaP}$ & $17(21.3)$ & $9(20.9)$ & & 2 & $22(27.5)$ & $18(4 \mid .9)$ & \\
\hline Age (years) & 71.25 (8.07) & $73.8(8)$ & 0.1 & 3 & $20(25)$ & $9(20.9)$ & \\
\hline Female sex & $4 \mid(5 I .3)$ & $19(44.2)$ & 0.57 & 4 & $13(16.3)$ & $4(9.3)$ & \\
\hline Hypertension & $69(86.3)$ & $37(86)$ & 0.99 & pWMLs & $1.53(0.98)$ & $1.62(0.85)$ & 0.71 \\
\hline Coronary artery disease & $18(22.5)$ & $6(14)$ & 0.34 & pWMLs 0-I & $45(55)$ & $23(54)$ & 0.5 \\
\hline Diabetes mellitus & $46(56.3)$ & $23(53.5)$ & 0.85 & $2-3$ & $35(45)$ & $20(46)$ & \\
\hline Current smoking & $25(3 \mid .3)$ & $12(27.9)$ & 0.83 & dWMLs & $\mathrm{I} .47(0.87)$ & $2.04(0.87)$ & 0.01 \\
\hline Hyperlipidemia & $62(77.5)$ & $29(67.4)$ & 0.28 & dWMLs 0-I & $46(56.7)$ & $21(49)$ & 0.07 \\
\hline Peripheral artery disease & $5(6.3)$ & $5(I 1.6)$ & 0.31 & $2-3$ & $34(43.7)$ & $22(5 \mathrm{I})$ & \\
\hline Polymetabolic syndrome & $37(46.3)$ & $16(37.2)$ & 0.34 & Lacunes & $42(52.5)$ & 18 (4I.9) & 0.34 \\
\hline Obesity (BMI>30 kg/m²) & $63(78.8)$ & $29(67.4)$ & 0.19 & Microbleeds & $14(17.5)$ & $5(11.6)$ & 0.44 \\
\hline Chronic kidney disease & $9(11.3)$ & $9(20.9)$ & 0.18 & PVS & $34(42.5)$ & $19(44.2)$ & 0.99 \\
\hline Use of statins & $64(80)$ & $32(74.4)$ & 0.2 & Atrophy & $20(25.3)$ & $13(30.2)$ & 0.67 \\
\hline Use of aspirin medications & $68(85)$ & $32(74.4)$ & 0.4 & MAP $(\mathrm{mmHg})$ & $93.1(11.7)$ & $97.3(12)$ & 0.15 \\
\hline
\end{tabular}

Notes: Values are mean $( \pm S D)$ or numbers of patients (\%); *Mann-Whitney $U$ test or chi-square test.

Abbreviations: SVD, small-vessel disease; VaD, vascular dementia; VaP, vascular parkinsonism; LS, lacunar stroke; BMI, body mass index; WMLs, white matter lesions; pWMLs, periventricular WMLs; dWMLs, deep WMLs; PVS, enlarged perivascular spaces; MAP, mean arterial pressure.

progression group) or the development of new lacunes (new lacunes group) in one or more periventricular and/or subcortical regions (Figure 1).

Detailed neurological examinations and neuropsychological evaluation were undertaken at the baseline and follow-up visits. All patients received optimal medical treatment, according to guidelines. Baseline risk factors including the average 24-hour mean arterial pressure (MAP) recorded by 24-hour ambulatory blood pressure monitoring (Schiller MT-300) and the concentrations of biochemical compounds were compared between patients with and without radiological progression. To avoid the problem of multiple comparisons, we used composite measures. For each patient, the mean $Z$-score combined blood markers specific for vascular or systemic inflammation. A $Z$-score was calculated as the (value in an individual minus the mean value in the study population)/ standard deviation. We used logistic regression to obtain the OR $(95 \% \mathrm{CI})$ for the association of each biomarker (highest vs lowest tertile) and each outcome (any progression of WMLs and/or development of new lacunes, development

Table 2 Comparison of biochemical markers between patients with and without radiological progression

\begin{tabular}{|c|c|c|c|c|c|c|c|}
\hline \multirow[t]{2}{*}{ Variable } & \multicolumn{3}{|c|}{ Any radiological progression } & \multicolumn{2}{|c|}{ New lacunes } & \multicolumn{2}{|c|}{ WML progression } \\
\hline & No & Yes & $p$-value & Yes & p-value* & Yes & $p$-value* \\
\hline N (\%) & $80(65)$ & $43(35)$ & & $10(23.2)$ & & $18(43)$ & \\
\hline Age (years) & 71.25 (8.07) & $73.8(8)$ & 0.1 & $72.90(8.8)$ & 0.55 & $72.78(7)$ & 0.46 \\
\hline hsCRP (mg/dL) & $0.68(1.9)$ & $0.85(1.2)$ & 0.6 & $0.67(1.01)$ & 0.98 & $0.5 I(0.9)$ & 0.72 \\
\hline PGL (mg/dL) & $121.86(43.5)$ & I 23.53 (49.8) & 0.84 & $135.7(70)$ & 0.38 & II3.6I (38) & 0.46 \\
\hline eGFR $\left(\mathrm{mL} / \mathrm{min} / \mathrm{I} .73 \mathrm{~m}^{2}\right)$ & $81.17(23.1)$ & $72.45(26)$ & 0.06 & $66.2(35.1)$ & 0.07 & $68.03(21.3)$ & 0.03 \\
\hline Homocysteine ( $\mu \mathrm{mol} / \mathrm{L})$ & $13.88(5.4)$ & $16.55(7.5)$ & 0.02 & $19.43(12.3)$ & 0.01 & $15.36(4.9)$ & 0.29 \\
\hline sP-selectin (ng/mL) & I58.4 (44.3) & $153.5(46.6)$ & 0.66 & $165.4(18.1)$ & 0.73 & $150.2(42.6)$ & 0.56 \\
\hline sICAM (ng/mL) & $314.4(109.8)$ & $308.5(89.8)$ & 0.79 & $270.9(5 \mathrm{I} .7)$ & 0.34 & $332.8(104.9)$ & 0.55 \\
\hline PF-4 (ng/mL) & $5.42(8.1)$ & $22.8(39)$ & $<0.01$ & $5.3(3.6)$ & 0.96 & $31.2(12)$ & $<0.01$ \\
\hline $\mathrm{IL}-\mathrm{I} \alpha(\mathrm{pg} / \mathrm{mL})$ & $0.70(3.06)$ & $\mathrm{I} .4(5.7)$ & 0.41 & $0.13(0.1)$ & 0.65 & $0.75(1.5)$ & 0.95 \\
\hline TNF- $\alpha(p g / m L)$ & $4.96(4.08)$ & $6.49(3.1)$ & 0.25 & $6.08(4.5)$ & 0.66 & $8.08(0.7)$ & 0.30 \\
\hline sCD40 L (ng/mL) & $6.9(4.53)$ & I0.I (3.6) & 0.03 & II.I3 (2.4) & 0.13 & $6.8(1.8)$ & 0.98 \\
\hline IL-6 (pg/mL) & $4.96(6.8)$ & $15.19(23.8)$ & 0.03 & $38.08(42.1)$ & $<0.0 \mathrm{I}$ & $2.5 I(3.1)$ & 0.62 \\
\hline Z-score for VI & $-0.08(0.45)$ & $0.15(0.54)$ & 0.01 & $0.14(0.48)$ & 0.1 & $0.16(0.57)$ & 0.02 \\
\hline Z-score for SI & $-0.07(0.43)$ & $0.14(0.66)$ & 0.03 & $0.26(0.84)$ & 0.02 & $0.1(0.53)$ & 0.19 \\
\hline
\end{tabular}

Notes: Values are mean \pm SD; Z-score for VI combined (sICAM-I, sCD40 L, sP-selectin, homocysteine, and PF-4); Z-score for SI combined (hsCRP, IL-I $\alpha$, IL-6, and TNF- $\alpha$ ). *Compared to patients without radiological progression.

Abbreviations: WMLs, white matter lesions; IL-I $\alpha$, interleukin I; IL-6, interleukin-6; TNF, tumor necrosis factor; hsCRP, high-sensitivity C-reactive protein; PGL, plasma glucose level; sCD40 L, soluble CD40 ligand; VI, vascular inflammation; SI, systemic inflammation; sICAM, soluble intercellular cell adhesion molecule-I; PF-4, platelet factor-4. 
Table 3 ORs for the association between baseline biomarkers and any radiological progression, new lacunes, or WML progression

\begin{tabular}{|c|c|c|c|c|c|c|c|c|c|}
\hline \multirow[t]{2}{*}{ Variable } & \multicolumn{6}{|c|}{ Any radiological progression } & \multicolumn{3}{|l|}{ New lacunes } \\
\hline & Unadjusted* & $p$-value & Model I & $p$-value & Model 2 & $p$-value & Unadjusted* & $p$-value & Model I \\
\hline TNF- $\alpha$ & $4.4(0.8-16.2)$ & 0.3 & & & & & $2.2(0.4-11.1)$ & 0.7 & \\
\hline sICAM-I & $1.08(0.3-4)$ & 0.9 & & & & & $0.5(0.15-1.9)$ & 0.3 & \\
\hline hsCRP & $0.56(0.16-1.9)$ & 0.35 & & & & & $0.6(0.1-6.3)$ & 0.7 & \\
\hline sP-selectin & $1.6(0.4-7.3)$ & 0.5 & & & & & $2(0.4-9.7)$ & 0.3 & \\
\hline IL-I $\alpha$ & $0.6(0.15-2.3)$ & 0.5 & & & & & $0.7(0.17-2.7)$ & 0.6 & \\
\hline Homocysteine & $4.1(1.1-15.4)$ & 0.03 & $4.1(0.99-17)$ & 0.05 & $3.9(0.93-16.8)$ & 0.06 & $3.1(1.7-9.2)$ & 0.02 & $2.4(1.4-14)$ \\
\hline PF-4 & $5.7(1.6-20.3)$ & 0.01 & $5.5(|.5-2|)$ & 0.01 & $6.9(1.7-28.1)$ & 0.01 & $3.1(0.5-16.5)$ & 0.9 & \\
\hline sCD40 L & $3.9(1.05-14.6)$ & 0.04 & $4.6(1.1-18.6)$ & 0.03 & $3.9(0.91-17.2)$ & 0.07 & $9.7(0.8-29.7)$ & 0.15 & \\
\hline IL-6 & $3.9(1.05-14.3)$ & 0.04 & $7.4(1.48-37)$ & 0.02 & $7.7(1.3-45.9)$ & 0.02 & $2.4(1.8-7.3)$ & 0.01 & $6(0.95-38)$ \\
\hline Z-score for VI & $4.5(1.1-17.7)$ & 0.02 & $4.5(1.1-18.6)$ & 0.03 & $4.3(1.02-17.9)$ & 0.04 & $2.2(0.76-10.7)$ & 0.13 & \\
\hline Z-score for SI & $1.6(1.05-5.4)$ & 0.04 & $1.6(0.98-6.1)$ & 0.07 & $1.4(0.9-5.7)$ & 0.1 & $2.7(1.5-12.9)$ & 0.03 & $2.1(1.2-14)$ \\
\hline
\end{tabular}

Notes: *Values are OR $(95 \% \mathrm{Cl})$ highest vs lowest tertile; Model I: adjusted for baseline MAP, SVD score, age, and sex; Model 2: adjusted additionally for clinical SVD manifestations; Z-score for VI combined (sICAM-I, sCD40 L, sP-selectin, homocysteine, and PF-4); Z-score for SI combined (hsCRP, IL-I $\alpha$, IL-6, and TNF- $\alpha$ ).

Abbreviations: sICAM-I, soluble intercellular cell adhesion molecule-I; WMLs, white matter lesions; IL-I $\alpha$, interleukin I; IL-6, interleukin-6; TNF, tumor necrosis factor; hsCRP, high-sensitivity C-reactive protein; sCD40 L, soluble CD40 ligand; VI, vascular inflammation; SI, systemic inflammation; PF-4, platelet factor-4; MAP, mean arterial pressure; SVD, small-vessel disease.

of new lacunes, and WML progression). Biomarkers that achieved a $p$-value $\leq 0.1$ were included in the multivariate regression model, which was adjusted for baseline MAP, SVD score, age, sex (Model 1), and additionally adjusted for SVD clinical manifestations (Model 2), selected based on clinical plausibility and previous literature reviews. Statistical significance was set at $p$-value $<0.05$ for all analyses.

The study complied with the Declaration of Helsinki. All participants signed an informed consent form. The study was approved by the local Medical Ethics Committee (Komisja Bioetyczna przy Wojskowym Instytucie Medycznym, 46/WIM/2010).

\section{Results}

The study population comprised 123 older adults (age, mean $\pm S D, 72.2 \pm 8$ years) of both sexes (48.8\% females) with symptomatic SVD. Patients with VaD, VaP, and LS differed in regard to their mean age $(74.2 \pm 7.9$ vs $73 \pm 5.9$ vs 69.6 \pm 8.6 , respectively, ANOVA $p=0.01$ ), the results of the SVD scale $(1.68 \pm 0.8 ; 1.9 \pm 1$; and $2.8 \pm 0.9 ; p=0.01)$, and female sex distribution ( $75 \%$ vs 34.6 vs $30.6 \%, \chi^{2} p=0.01$ ); however, there were no other discrepancies among the prevalence values of vascular risk factors nor among the levels of the studied biomarkers. Forty-three patients $(35 \%)$ experienced radiological progression; the evolution of WMLs was observed in $43 \%(n=18)$ of these cases, new lacunes in $23.2 \%(n=10)$, and both WML progression and new lacunes in $34.8 \%(n=15)$. Among patients who had radiological progression, 21 patients (48.8\%) had clinically evident LS, 17 patients $(39.5 \%)$ experienced deterioration in cognitive function, and all subjects had either relevant LI or WML progression on imaging. Although the mean SVD scores were similar, more than half of the cases that progressed had a higher prevalence of dWMLs or VaD (Table 1). No other variations in the distribution of vascular risk factors were found. Patients with any level of radiological progression had elevated levels of homocysteine, sCD40 L, PF-4, and IL-6, which resulted in higher Z-scores for VI and SI; patients with isolated new lacunes had elevated homocysteine, IL-6, and $Z$-scores for SI; and those with WML progression had elevated PF-4 and Z-scores for VI compared to patients without progression (Table 2).

After adjusting for confounders (baseline MAP, SVD score, age, and sex) in the multivariate analysis, the PF-4, sCD40 L, IL-6, Z-score for VI and, marginally, homocysteine, and $Z$-score for SI were associated with the risk of any radiological progression; homocysteine, $Z$-score for SI and, marginally, IL-6 were related to the development of new lacunes, and PF-4 and marginally $Z$-score for VI were correlated with the risk of WML progression (Table 3). Additional adjustment for clinical SVD manifestations did not significantly alter the abovementioned findings.

\section{Discussion}

Our study demonstrated that both WMLs and lacunes progressed over 2 years, according to the follow-up data, in more than one-third of older adults with symptomatic SVD. The principal finding was that, in a panel of different biomarkers, PF-4, sCD40 L, homocysteine, and IL-6 were each associated with any level of radiological progression, independent of age, sex, baseline blood pressure, and MRI markers of SVD. However, the strength of the associations differed among the radiological determinants of SVD. Higher levels of homocysteine and, marginally, of IL-6 predicted the development of new lacunes only, and WML progression was associated with elevated PF-4. Although both Z-scores were associated 


\begin{tabular}{|c|c|c|c|c|c|c|c|c|}
\hline \multirow[b]{2}{*}{$p$-value } & \multirow[b]{2}{*}{ Model 2} & \multirow[b]{2}{*}{$p$-value } & \multicolumn{6}{|c|}{ WML progression } \\
\hline & & & Unadjusted* & $p$-value & Model I & $p$-value & Model 2 & $p$-value \\
\hline & & & $2.4(0.65-8.6)$ & 0.32 & & & & \\
\hline & & & $1.9(0.6-5.9)$ & 0.2 & & & & \\
\hline & & & $1.5(0.6-4.3)$ & 0.35 & & & & \\
\hline & & & I.I (0.3-3.9) & 0.8 & & & & \\
\hline & & & $0.7(0.2-2.1)$ & 0.7 & & & & \\
\hline \multirow[t]{3}{*}{0.03} & $1.9(1.3-12.7)$ & 0.04 & $2.3(0.7-7.2)$ & 0.16 & & & & \\
\hline & & & $2.7(1.06-6.9)$ & 0.02 & $7.9(1.6-38)$ & 0.01 & $12.4(2 .|-7|)$ & 0.01 \\
\hline & & & $1.4(0.3-7)$ & 0.6 & & & & \\
\hline \multirow[t]{2}{*}{0.05} & $6.1(0.7-50)$ & 0.09 & $1.9(0.7-5.2)$ & 0.2 & & & & \\
\hline & & & $2.6(1.03-7.1)$ & 0.04 & $4.2(0.9-19.5)$ & 0.06 & $4.4(0.88-22)$ & 0.07 \\
\hline 0.04 & $2.1(1.2-15)$ & 0.04 & I.4 (0.5-3.7) & 0.4 & & & & \\
\hline
\end{tabular}

with any radiological progression in univariate analysis, after adjusting for confounders, the $Z$-score representing systemic inflammation markers was significantly associated only with the risk of new lacunes, whereas the $Z$-score for vascular inflammation was merely marginally related to the risk of WML progression. Interestingly, these associations did not change after adjusting for SVD clinical manifestations.

Several longitudinal studies documented that the most consistent predictor for progression of WMLs is the baseline severity of WMLs and that radiological markers have better predictive value with regard to long-term functional outcome as compared to plasma markers. ${ }^{12}$ Furthermore, a meta-analysis and systematic review of 46 prospective studies revealed the doubled risk of death among patients with WMLs in comparison to those without WMLs. ${ }^{13}$ However, only limited data are available on the relationship between biomarkers and the radiological progression of SVD, which results in our data being more important. Our results supported those from the SMART-MR study, which found an association between homocysteine levels and radiological progression. In contrast, some studies found associations between serum ICAM-1 and CRP levels, whereas - in a 3C Dijon study - no significant relations between baseline CRP or IL-6 levels and MRI changes were observed. However, these studies included mainly asymptomatic patients and had longer follow-up periods (4-6 years). ${ }^{14-16}$ The association among PF-4, sCD40 L, and radiological progression is a new finding, and, to our knowledge, this association has not been previously reported. Moreover, the relationship between plasma markers and clinical progression in SVD is not clear. In the LIMITS study, an association between IL-6 and TNF- $\alpha$ and recurrent cerebrovascular events following LS was demonstrated; however, sCD40 L and serum amyloid
A were not related to that risk. ${ }^{17}$ By contrast, various studies suggested that SCD40 L and PF-4 were associated with risk of vascular events, infarct size, and worse clinical courses in stroke. ${ }^{18,19}$ Although the majority of patients in our cohort that had radiological progression experienced LS or had cognitive decline, we did not focus on clinical outcome in the current analysis because this issue will be addressed in further studies. ${ }^{5}$

Our findings supported a complex interaction between pro-inflammatory mechanisms and platelet activation in SVD that may result in ED and dysfunction of the neurovascular unit. ${ }^{20} \mathrm{~A}$ number of experimental studies showed that white matter is particularly vulnerable to inflammatory mediators which contribute to the development of brain lesions and neurological deficits in SVD. ${ }^{21}$ Increase in the permeability of the blood-brain barrier (BBB) in SVD probably induces a series of acute or chronic events leading to oxidative stress and inflammatory mechanisms that promoting ED itself as well as cumulative inflammatory cell infiltration resulting in local or global brain damage. ${ }^{22}$ However, the cause of the BBB dysfunction and inflammation - whether indicating nonspecific responses to vascular risk factors, peripheral systemic stimuli, systemic inflammation, or brain specific is not known. ${ }^{23}$ Diverse radiological features of SVD that probably reflect different pathological mechanisms or disease stages make the interpretation of experimental data challenging. Lacunes are thought to represent the focal manifestation, whereas WMLs denote a diffuse abnormality in the small arterioles, resulting in a state of chronic hypoperfusion of the white matter in the brain. ${ }^{23}$ Indeed, our study showed diverse correlations between markers of radiological progression and combined $Z$-scores for systemic and vascular inflammation. Our data requires replication to ensure validity, 
but, if validated, it lends support to the involvement of various components of the inflammatory cascade in the pathogenesis of WMLs and LIs. However, as SVD radiological markers frequently coexist, it is possible they share common intrinsic microvascular pathologies and, therefore, owing to its diffuse nature, SVD should be regarded as a whole-brain disease. $^{24}$

Currently, there is no blood biomarker that fulfils the criteria of a surrogate endpoint in SVD; however, those representing ED may prove useful in defining SVD heterogeneity, especially at early stages of the disease, and, in combination with neuroimaging markers, they may show better correlation with clinical outcomes. Although the management of traditional risk factors and antiplatelet treatment (for acute LS) remain the most important preventive approach in SVD, increasing evidence suggests that new studies should consider drugs that target the endothelium and BBB to prevent and treat SVD. ${ }^{17}$

There were several limitations to our study. The small number of patients may have produced biased estimates, and the results may not be generalizable to other populations and should be considered exploratory in nature; however, this is also a limiting factor in most published reports on this subject. Multiple correlations could result in Type I error but calculating the $Z$-score eliminates some issues raised by multiple testing of single markers. Another weakness was the visual assessment of the imaging; however, the SVD and Fazekas scales remain the most widely used in the research literature. This study was, therefore, regarded as hypothesis-generating rather than definitive, and larger studies and replications are needed for more robust conclusions. Although patients with chronic inflammatory diseases were not included in the study, blood biomarkers analyzed in the present study can be either brain-specific or systemic markers. On the other hand, our study had some advantages. We studied a wellphenotyped group of patients with SVD that included VaP and $\mathrm{VaD}$ subjects, and we analyzed a broad set of biomarkers and their associations with the different imaging markers of SVD progression.

\section{Conclusion}

We showed that ED is not only a vascular disease marker but plays a role in the radiological progression of SVD; the concentrations of IL-6, PF-4, and sCD40 L were each independently associated with an increased risk of any level of radiological progression, as expressed by new lacunes or WML progression. In addition, the two radiological subtypes of SVD demonstrated differences in other associated biochemical markers. Z-scores for systemic inflammation, IL-6 and homocysteine levels predicted the development of new lacunes, and the $Z$-scores for vascular inflammation and PF-4 were associated with the risk of WML progression. Further research is required to ensure the validity of our results.

\section{Acknowledgment}

The study was supported by the Polish Ministry of Science and Higher Education as a research project (grant no N N402 473840).

\section{Disclosure}

The authors report no conflicts of interest in this work.

\section{References}

1. Pantoni L. Cerebral small vessel disease: from pathogenesis and clinical characteristics to therapeutic challenges. Lancet Neurol. 2010;9(7): 689-701.

2. Wardlaw JM, Smith C, Dichgans M. Mechanisms underlying sporadic cerebral small vessel disease: insights from neuroimaging. Lancet Neurol. 2013;12(5):70060-70067.

3. Wardlaw JM, Allerhand M, Doubal FN, et al. Vascular risk factors, large-artery atheroma, and brain white matter hyperintensities. Neurology. 2014;82(15):1331-1338.

4. Staszewski J, Piusińska-Macoch R, Brodacki B, Skrobowska E, Stępień A. Association between hemostatic markers, serum lipid fractions and progression of cerebral small vessel disease: a 2-year follow-up study. Neurol Neurochir Pol. 2018;52(1):54-63.

5. Staszewski J, Piusińska-Macoch R, Skrobowska E, et al. Significance of haemodynamic and haemostatic factors in the course of different manifestations of cerebral small vessel disease: the SHEF-SVD StudyStudy Rationale and Protocol. Neurosci J. 2013;2013:424695.

6. Bamford J, Sandercock P, Dennis M, Burn J, Warlow C. Classification and natural history of clinically identifiable subtypes of cerebral infarction. Lancet. 1991;337:1521-1526.

7. Hurtig HI. Vascular parkinsonism. In: Stern MB, Koller WC, editors. Parkinsonian Syndromes. New York: Marcel Dekker; 1993:81-83.

8. Roman GC, Tatemichi TK, Erkinjuntti T, et al. Vascular dementia: diagnostic criteria for research studies: report of the NINDS-AIREN International Workshop. Neurology. 1993;43:250-260.

9. Shoamanesh A, Preis SR, Beiser AS, et al. Inflammatory biomarkers, cerebral microbleeds, and small vessel disease: Framingham Heart Study. Neurology. 2015;84(8):825-832.

10. Wardlaw JM, Smith EE, Biessels GJ, et al; STandards for ReportIng Vascular changes on nEuroimaging (STRIVE v1). Neuroimaging standards for research into small vessel disease and its contribution to ageing and neurodegeneration. Lancet Neurol. 2013;12(8):822-838.

11. Staals J, Makin SD, Doubal FN, Dennis MS, Wardlaw JM. Stroke subtype, vascular risk factors, and total MRI brain small-vessel disease burden. Neurology. 2014;83(14):1228-1234.

12. Protopsaltis J, Kokkoris S, Korantzopoulos P, et al. Prediction of longterm functional outcome in patients with acute ischemic non-embolic stroke. Atherosclerosis. 2009;203(1):228-235.

13. Debette S, Markus HS. The clinical importance of white matter hyperintensities on brain magnetic resonance imaging: systematic review and metanalysis. BMJ. 2010;341:c3666. 
14. Umemura T, Kawamura T, Umegaki H, et al. Endothelial and inflammatory markers in relation to progression of ischaemic cerebral smallvessel disease and cognitive impairment: a 6-year longitudinal study in patients with type 2 diabetes mellitus. J Neurol Neurosurg Psychiatry. 2011;82(11):1186-1194.

15. Markus HS, Hunt B, Palmer K, Enzinger C, Schmidt H, Schmidt R. Markers of endothelial and hemostatic activation and progression of cerebral white matter hyperintensities: longitudinal results of the Austrian Stroke Prevention Study. Stroke. 2005;36(7):1410-1414.

16. Satizabal CL, Zhu YC, Mazoyer B, Dufouil C, Tzourio C. Circulating IL-6 and CRP are associated with MRI findings in the elderly: the 3C-Dijon Study. Neurology. 2012;78(10):720-727.

17. Boehme AK, McClure LA, Zhang Y, et al. Inflammatory markers and outcomes after lacunar stroke: the levels of inflammatory markers in treatment of stroke study. Stroke. 2016;47(3):659-667.

18. Novo S, Basili S, Tantillo R, et al. Soluble CD40L and cardiovascular risk in asymptomatic low-grade carotid stenosis. Stroke. 2005;36(3):673-675.
19. Welsh P, Barber M, Langhorne P, Rumley A, Lowe GD, Stott DJ. Associations of inflammatory and haemostatic biomarkers with poor outcome in acute ischaemic stroke. Cerebrovasc Dis. 2009;27(3):247-253.

20. Maas MB, Furie KL. Molecular biomarkers in stroke diagnosis and prognosis. Biomark Med. 2009;3(4):363-383.

21. Kaiser D, Weise G, Moller K, et al. Spontaneous white matter damage, cognitive decline and neuroinflammation in middle-aged hypertensive rats: an animal model of early-stage cerebral small vessel disease. Acta Neuropathol Commun. 2014;2:169.

22. Fu Y, Yan Y. Emerging role of immunity in cerebral small vessel disease. Front Immunol. 2018;9:67.

23. Wardlaw JM. What causes lacunar stroke? J Neur Neurosurg Psych. 2005;76(5):617-619.

24. Shi Y, Wardlaw JM. Update on cerebral small vessel disease: a dynamic whole-brain disease. Stroke Vasc Neurol. 2016;1(3):83-92.
Clinical Interventions in Aging

\section{Publish your work in this journal}

Clinical Interventions in Aging is an international, peer-reviewed journal focusing on evidence-based reports on the value or lack thereof of treatments intended to prevent or delay the onset of maladaptive correlates of aging in human beings. This journal is indexed on PubMed Central, MedLine,

\section{Dovepress}

CAS, Scopus and the Elsevier Bibliographic databases. The manuscript management system is completely online and includes a very quick and fair peer-review system, which is all easy to use. Visit http://www.dovepress. com/testimonials.php to read real quotes from published authors. 